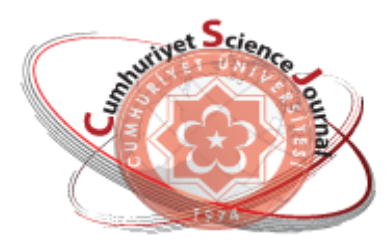

e-ISSN: 2587-246X

ISSN: $2587-2680$

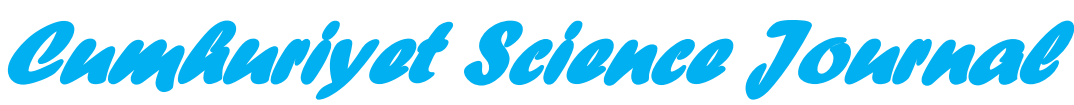

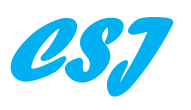

Cumhuriyet Sci. J., Vol.38-3 (2017) 473-479

\title{
Semiprime Rings with Multiplicative Generalized Derivations on Lie Ideals
}

\author{
Emine $\mathrm{KOÇ}$ \\ Cumhuriyet University, Faculty of Science, Department of Mathematics, 58140 Sivas, TURKEY
}

Received: 15.05.2017; Accepted: 05.06.2017

http://dx.doi.org/10.17776/csj.340488

\begin{abstract}
Let $R$ be a 2 - torsion free semiprime ring. In [10], a map $F: R \rightarrow R$ is called a multiplicative generalized derivation if there exists a map $d: R \rightarrow R$ such that $F(x y)=F(x) y+x d(y)$, for all $x, y \in R$. Let $U$ be a noncentral square-closed Lie ideal of $R$ and $F, G$ multiplicative generalized derivations associated to the maps $d, h$ of $R$ respectively such that $d(U) \subseteq U$ and $F(2 x)=2 F(x), G(2 x)=2 G(x)$ for all $x \in R$. In the present paper, we shall prove that $d$ is commuting map on $U$ if any one of the following holds: i) $G(u v)+$ $d(u) F(v) \pm u v \in Z$, ii) $G(u v)+d(u) F(v) \pm v u \in Z$. If any one of the conditions iii) $F([u, v])=$ $\pm[G(u), G(v)]$, and iv) $F(u \circ v)= \pm(G(u) \circ G(v))$, for all $u, v \in U$ are satisfied, then $[U, U] U h(Z \cap U)=$ $(0)$.
\end{abstract}

Keywords: Semiprime ring, multiplicative generalized derivation, generalized derivation, Lie ideal.

\section{Lie İdealler Üzerinde Çarpımsal Genelleştirilmiş Türevli Yarıasal Halkalar}

\begin{abstract}
Özet. $R, 2$-torsion free bir yarıasal halka olsun. [10] dan, eğer her $x, y \in R$ için $F(x y)=F(x) y+x d(y)$ koşulunu sağlayan bir $d: R \rightarrow R$ dönüşümü varsa $F$ dönüşümüne $R$ halkasının $d$ ile belirlenmiş bir çarpımsal genelleştirilmiş türevi denir. $U, R$ halkasının bir merkez tarafından kapsanılmayan kare-kapalı Lie ideali, $F, G$ dönüşümleri $R$ halkasının sırasıyla $h$ ve $d$ dönüşümleri ile belirlenmiş çarpımsal genelleştirilmiş türevleri, $d(U) \subseteq U$ ve her $x \in R$ için $F(2 x)=2 F(x), G(2 x)=2 G(x)$ olsun. Bu çalışmada, aşağıdaki koşullardan biri sağlanırsa $d$, dönüşümünün $U$ üzerinde komüting dönüşüm olduğu gösterilecektir: i) $G(u v)+d(u) F(v) \pm$ $u v \in Z$, ii) $G(u v)+d(u) F(v) \pm v u \in Z$. Ayrıca her $u, v \in U$ için iii) $F([u, v])= \pm[G(u), G(v)]$, iv) $F(u \circ v)= \pm(G(u) \circ G(v))$ koşullarından biri sağlanırsa bu durumda $[U, U] \operatorname{Uh}(Z \cap U)=(0)$ olduğu ispatlanacaktır.
\end{abstract}

Anahtar Kelimeler: Yarıasal halka, çarpımsal genelleştirilmiş türev, genelleştirilmiş türev, Lie ideal.

\section{INTRODUCTION}

Throughout $R$ will present an associative ring with center $Z$. For any $x, y \in R$, the symbol $[x, y]$ stands for the commutator $x y-y x$ and the symbol $x \circ y$ denotes the anti-commutator $x y+y x$. Recall that a ring $R$ is prime if for $x, y \in R, x R y=(0)$ implies $x=0$ or $y=0$, and $R$ is semiprime if for $x \in$ $R, x R x=(0)$ implies $x=0$. An additive subgroup $U$ of $R$ is said to be a Lie ideal of $R$ if $[u, r] \in U$, for all $u \in U, r \in R$. $U$ is called a square-closed Lie ideal of $R$ if $U$ is a Lie ideal and $u^{2} \in U$ for all $u \in$ $U$. Let $S$ be a nonempty subset of $R$. A mapping $F$ from $R$ to $R$ is called centralizing on $S$ if $[F(x), x] \in Z$, for all $x \in S$ and is called commuting on $S$ if $[F(x), x]=0$, for all $x \in S$. A mapping $F$ from $R$ to $R$ is called strong commutativity preserving (simply, SCP) on $S$ if $[x, y]=[F(x), F(y)]$ for all $x, y \in S$. 
An additive mapping $d: R \rightarrow R$ is called a derivation if $d(x y)=d(x) y+x d(y)$ holds for all $x, y \in R$. This observation leads to the following definition given by Bresar in [7]. An additive mapping $F: R \rightarrow R$ is called a generalized derivation if there exists a derivation $d: R \rightarrow R$ such that

$$
F(x y)=F(x) y+x d(y), \text { for all } x, y \in R .
$$

The commutativity of prime or semiprime rings with derivation was initiated by Posner [15]. Thereafter, several authors have proved commutativity theorems of prime or semiprime rings with derivations. The notion of multiplicative derivation was introduced by Daif [8] motivated by Martindale [14]. $d: R \rightarrow R$ is called a multiplicative derivation if $d(x y)=d(x) y+x d(y)$ holds for all $x, y \in R$. These maps are not additive. In [12], Goldman and Semrl gave the complete description of these maps. We have $R=C[0,1]$, the ring of all continuous (real or complex valued) functions and define a map $d: R \rightarrow R$ such as

$$
d(f)(x)=\left\{\begin{array}{cl}
\mathrm{f}(\mathrm{x}) \log |\mathrm{f}(\mathrm{x})|, & \mathrm{f}(\mathrm{x}) \neq 0 \\
0, & \text { otherwise }
\end{array}\right.
$$

It is clear that $\mathrm{d}$ is multiplicative derivation, but $\mathrm{d}$ is not additive. In [9], inspired by the definition multiplicative derivation, the notion of multiplicative generalized derivation was extended by Daif and Tamman El-Sayiad as follows:

$F: R \rightarrow R$ is called a multiplicative generalized derivation if there exists a derivation $d: R \rightarrow R$ such that $F(x y)=F(x) y+x d(y)$, for all $x, y \in$ $R$. Dhara and Ali gave a slight generalization of this definition taking $d$ is any map (not necessarily an additive map or a derivation) in [10]. Every generalized derivation is a multiplicative generalized derivation. But the converse is not ture in general. In [2], Ashraf and Rehman showed that a prime ring $R$ with a nonzero ideal $I$ must be commutative if it admits a derivation $d$ satisfying either of the properties $d(x y)+x y \in Z$ or $d(x y)-x y \in Z$ for all $x, y \in R$. In [3], the authors explored the commutativity of prime ring
$\mathrm{R}$ in which satisfies any one of the properties when $\mathrm{f}$ is a generalized derivation. In [17], studied the commutativity of such a prime ring if anyone of the following is central: $G(x y)+F(x) F(y) \pm x y=0$ or $\quad G(x y)+F(x) F(y) \pm y x=0 \quad$ where $(F, d),(G, h)$ are generalized derivations. In the present paper, we shall extend the above results for a noncentral square-closed Lie ideal of semiprime rings with $F, G$ multiplicative generalized derivations associated to the derivations $d, h$ of $R$ respectively such that $d(U) \subseteq U$ and $F(2 x)=$ $2 F(x), G(2 x)=2 G(x)$ for all $x \in R$.

There is also a growing literature strong commutativity preserving (SCP) maps and derivations (for reference see [5] and [11]). In [1], Ali, Yasen and Anwar showed that if $R$ is a semiprime ring, $f$ is an endomorphism which is a strong commutativity preserving (simply, SCP) map on a nonzero ideal $U$ of $R$, then $f$ is commuting on $U$. Derivations as well as SCP mappings have been extensively studied by researchers in the context of operator algebras, prime rings and semiprime rings too. In this paper, we extend some well known these results concerning of semiprime rings to a multiplicative generalized derivation.

Throughout the present paper, we shall make use of the following basic identities without any specific mention:

$$
\begin{array}{cl}
\text { i. } & {[x, y z]=y[x, z]+[x, y] z} \\
\text { ii. } & {[x y, z]=x[y, z]+[x, z] y} \\
\text { iii. } & x \circ(y z)=(x \circ y) z-y[x, z]= \\
& y(x \circ z)+[x, y] z \\
\text { iv. } & (x y) \circ z=x(y \circ z)-[x, z] y= \\
& (x \circ z) y+x[y, z]
\end{array}
$$

\section{RESULTS}

Lemma 2.1. [16, Lemma 2] Let $R$ be a 2 -torsion free semiprime ring, $U$ a noncentral Lie ideal of $R$ and $a \in U$. If $a U a=0$, then $a^{2}=0$ and there exists a nonzero ideal $K=R[U, U] R$ of $R$ generated by $[U, U]$ such that $[K, R] \subseteq U$ and $K a=$ $a K=0$. 
Corollary 2.2. [13, Corollary 3] Let $R$ be a 2 -torsion free semiprime ring, $U$ a noncentral Lie ideal of $R$ and $a, b \in U$.

(i) If $a U a=(0)$, then $a=0$.

(ii) If $a U=(0)$ ( or $U a=(0))$, then $a=0$.

(iii) If $U$ is square-closed and $a U b=(0)$, then $a b=0$ and $b a=0$.

Lemma 2.3. [4, Theorem 7] Let $R$ be a prime ring with characteristic not two and $U$ a nonzero Lie ideal of $R$. If $d$ is a nonzero derivation of $R$ such that $[u, d(u)] \in Z$ for all $u \in U$, then $U \subseteq Z$.

Lemma 2.4. [6, Lemma 4] Let $R$ be a prime ring with characteristic not two, $a, b \in R$. If $U$ a noncentral Lie ideal of $R$ and $a U b=(0)$, then $a=0$ or $b=0$.

Throughout this paper, $R$ will be a 2 -torsion free semiprime ring admitting two multiplicative generalized derivations $F$ and $G, U$ a square-closed Lie ideal of $R$ such that $F(2 x)=2 F(x), G(2 x)=$ $2 G(x)$ for all $x \in R$.

Theorem 2.5. Let $R$ be a 2-torsion free semiprime ring, $U$ a noncentral square-closed Lie ideal of $R$ and $F, G$ multiplicative generalized derivations associated to the derivations $d, h$ of $R$ respectively such that $d(U) \subseteq U$. If $G(u v)+d(u) F(v) \pm$ $u v \in Z$, for all $u, v \in U$, then $d$ is commuting map on $U$.

Proof. By the hypothesis, we have

$G(u v)+d(u) F(v) \pm u v \in Z$ for all $u, v \in U$

Replacing $v$ by $2 v w, w \in U$ in (2.1), we have

$$
\begin{aligned}
& 2(G(u v) w+u v h(w)+d(u) F(v) w+ \\
& d(u) v d(w) \pm u v w) \in Z .
\end{aligned}
$$

Commuting this term with $w$, using the hypothesis and $R$ is 2-torsion free, we find

$$
[u v h(w), w]+[d(u) v d(w), w]=0 .
$$

Taking $u$ by $2 u w$ in (2.3), we get $[u w v h(w), w]+[d(u) w v d(w), w]+$ $[u d(w) v d(w), w]=0$.

Replacing $v$ by $2 w v$ in (2.3), we obtain that

$[u w v h(w), w]+[d(u) w v d(w), w]=0$.

Subtracting (2.5) from (2.4), we obtain

$[u d(w) v d(w), w]=0$, for all $u, v, w \in U$.

Replacing $u$ by $8 d(w) v d(w) u$ in (2.6) and using equation (2.6), we get

$[d(w) v d(w), w] u d(w) v d(w)=0$.

Replacing $u$ by $2 u w$ in (2.7), we get

$[d(w) v d(w), w] u w d(w) v d(w)=0$.

Right multiplying by $w$ in (2.7), we obtain

$[d(w) v d(w), w] u d(w) v d(w) w=0$.

Subtracting the last equation from (2.8), we have

$[d(w) v d(w), w] U[d(w) v d(w), w]=(0)$,

for all $v, w \in U$.

Using Corollary 2.2 and $R$ is 2-torsion free, we get

$[d(w) v d(w), w]=0$, for all $v, w \in U$,

and so

$d(w) v d(w) w-w d(w) v d(w)=0$.

Replacing $v$ by $4 v d(w) t, t \in U$ and since $R$ is 2torsion free, we obtain

$d(w) v d(w) t d(w) w-w d(w) v d(w) t d(w)=0$.

Using equation (2.10), we have

$d(w) v[w, d(w)] t d(w)=0$, for all $v, w, t \in U .(2.11)$

Replacing $v$ by $2 w v$ in this equation and using $R$ is 2-torsion free, we have

$d(w) w v[w, d(w)] t d(w)=0$, for all $v, w, t \in U$. 
Left multiplying by $w$ in (2.11), we obtain

$w d(w) v[w, d(w)] t d(w)=0$, for all $v, w, t \in U$.

Subtracting the last equation from above equation, we have

$[w, d(w)] v[w, d(w)] t d(w)=0$, for all $v, w, t \in U$.

Again, replacing $t$ by $2 t w$ in this equation and using $R$ is 2-torsion free, we have

$[w, d(w)] v[w, d(w)] t w d(w)=0$, for all $v, w, t \in U$.

Right multiplying by $w$ in the above equation, we obtain

$[w, d(w)] v[w, d(w)] t d(w) w=0$, for all $v, w, t \in U$.

Combining the last two equations, we have

$[w, d(w)] v[w, d(w)] t[w, d(w)]=0$, for all $v, w, t \in$ $U$.

Right multiplying this equation by $v[w, d(w)]$, we get

$[w, d(w)] v[w, d(w)] U[w, d(w)] v[w, d(w)]=(0)$, for all $u, v, w, t \in U$.

By Corollary 2.2 and since $R$ is 2-torsion free, we obtain

$[w, d(w)] v[w, d(w)]=0$, for all $v, w \in U$.

That is, $[w, d(w)]=0$, for all $w \in U$ by Corollary 2.2. Hence, $d$ is commuting on $U$. This completes the proof.

Corollary 2.6. Let $R$ be a 2-torsion free semiprime ring, $U$ a noncentral square-closed Lie ideal of $R$ and $F, G$ generalized derivations associated to the derivations $d, h$ of $R$ respectively such that $d(U) \subseteq$ $U$. If $G(u v)+d(u) F(v) \pm u v \in Z$, for all $u, v \in U$, then $d$ is commuting map on $U$.

Corollary 2.7. Let $R$ be a 2-torsion free prime ring, $U$ a square-closed Lie ideal of $R$ and $F, G$ generalized derivations associated to the derivations $d, h$ of $R$ respectively such that $d(U) \subseteq$ $U$. If $G(u v)+d(u) F(v) \pm u v \in Z$, for all $u, v \in U$, then $U \subseteq Z$.
Proof. Using the same arguments in the proof of Theorem 2.5, we get $d$ is commuting map on $U$. Using Lemma 2.3 and Lemma 2.4, we conclude that $U \subseteq Z$.

Corollary 2.8. Let $R$ be a 2-torsion free prime ring and $U$ a nonzero square-closed Lie ideal of $R$. If $R$ admits a generalized derivation $(F, d)$ such that $G(u v) \pm u v \in Z$ for all $u, v \in U$, then $U \subseteq Z$.

Proof. The proof is clear by Corollary 2.7 when $F=0$.

Theorem 2.9. Let $R$ be a 2-torsion free semiprime ring, $U$ a noncentral square-closed Lie ideal of $R$ and $F, G$ multiplicative generalized derivations associated to the derivations $d, h$ of $R$ respectively such that $d(U) \subseteq U$. If $G(u v)+d(u) F(v) \pm$ $v u \in Z$, for all $u, v \in U$, then $d$ is commuting map on $U$.

Proof. By the hypothesis, we have

$G(u v)+d(u) F(v)+v u \in Z$.

Replacing $v$ by $2 v w$ in the hypothesis, we obtain

$2(G(u v) w+u v h(w)+d(u) F(v) w+$

$d(u) v d(w)+v w u) \in Z$.

Commuting this term with $w$ and using $R$ is 2torsion free, we obtain

$[u v h(w), w]+[d(u) v d(w), w]+$ $[v[w, u], w]=0$.

Taking $u$ by $2 u w$ in (2.15) and since $R$ is 2-torsion free, we get

$[u w v h(w), w]+[d(u) w v d(w), w]+$ $[u d(w) v d(w), w]+[v[w, u] w, w]=0$.

Replacing $v$ by $2 w v$ in (2.15), we get

$[u w v h(w), w]+[d(u) w v d(w), w]+$ $[w v[w, u], w]=0$. 
Subtracting the above equation from (2.16), we find

$[u d(w) v d(w), w]+[[v[w, u], w], w]=0$.

Replacing $u$ by $2 u w$ in (2.17), we get

$[u w d(w) v d(w), w]+[[v[w, u], w], w] w=0$

Right multiplying by $w$ in (2.17) and then subtracting from (2.18), we obtain

$[u[d(w) v d(w), w], w]=0$

Replacing $u$ by $8 d(w) v d(w) u$ in (2.19), using Corollary 2.2 and $R$ is 2-torsion free, we obtain

$[d(w) v d(w), w]=0$, for all $w, v \in U$

This equation is the same as (2.10) in the proof of Theorem 2.5. Hence, using the same arguments in there, we get the required results. It is proved analogously using $G(u v)+d(u) F(v)-v u \in$ $Z(R)$, for all $u, v \in U$.

Corollary 2.10. Let $R$ be a 2-torsion free semiprime ring, $U$ a noncentral square-closed Lie ideal of $R$ and $F, G$ generalized derivations associated to the derivations $d, h$ of $R$ respectively such that $d(U) \subseteq U$. If $G(u v)+d(u) F(v) \pm$ $v u \in Z$, for all $u, v \in U$, then $d$ is commuting map on $U$.

Corollary 2.11. Let $R$ be a 2-torsion free prime ring, $U$ a square-closed Lie ideal of $R$ and $F, G$ generalized derivations associated to the derivations $d, h$ of $R$ respectively such that $d(U) \subseteq$ $U$. If $G(u v)+d(u) F(v) \pm v u \in Z$, for all $u, v \in U$, then $U \subseteq Z$.

Proof. Using the same arguments in the proof of Theorem 2.9, we get $d$ is commuting map on $U$. Using Lemma 2.3 and Lemma 2.4, we conclude that $U \subseteq Z$.

Corollary 2.12. Let $R$ be a 2-torsion free prime ring and $U$ a nonzero square-closed Lie ideal of $R$. If $R$ admits a generalized derivation $(F, d)$ such that $G(u v) \pm v u \in Z$ for all $u, v \in U$, then $U \subseteq Z$.
Proof. The proof is clear by Corollary 2.11 when $F=0$.

Theorem 2.13. Let $R$ be a 2-torsion free semiprime ring, $U$ a noncentral square-closed Lie ideal of $\mathrm{R}$ and $F, G$ multiplicative generalized derivations associated to the maps $d, h$ of $R$ respectively. If $F([u, v])= \pm[G(u), G(v)]$, for all $u, v \in U$, then $[U, U] U h(Z \cap U)=(0)$.

Proof. By the hypothesis, we have $F([u, v])= \pm[G(u), G(v)]$, for all $u, v \in U$

Replacing $v$ by $2 v z, z \in Z \cap U$ in the last equation and using $R$ is 2 -torsion free, we get

$$
\begin{gathered}
\begin{aligned}
0=F([u, v z]) & \pm[G(u), G(v z)] \\
& =F([u, v] z) \pm[G(u), G(v) z \\
& +v h(z)]
\end{aligned} \\
=F([u, v] z) \pm[G(u), G(v)] z \pm v[G(u), h(z)] \\
\pm[G(u), v] h(z)
\end{gathered}
$$

Using the hypothesis, we obtain

$[u, v] d(z) \pm v[G(u), h(z)] \pm[G(u), v] h(z)=0$ for all $u, v \in U$.

Replacing $v$ by $2 w v, w \in U$ in the above equation, we get

$w[u, v] d(z)+[u, w] v d(z) \pm w v[G(u), h(z)] \pm$ $w[G(u), v] h(z) \pm[G(u), w] v h(z)=0$.

Using equation (2.20), we obtain

$[u, w] v d(z) \pm[G(u), w] v h(z)=0$, for all

$u, v, w \in U$.

Replacing $u$ by $2 u z$ in the last equation, we have

$z[u, w] v d(z) \pm z[G(u), w] v h(z)+$

$[u h(z), w] v h(z)=0$, for all $u, v, w \in U$.

By equation (2.21), we find

$[u h(z), w] v h(z)=0$, for all $u, v, w \in U$. 
Replacing $v$ by $2 v u$ in the above relation and left multiplying this equation by $w$, we get

$[u h(z), w] v u h(z) w=0$, for all $u, v, w \in U$.

Substituting $v$ by $4 v w u$ in (2.22), we obtain

$[u h(z), w] \operatorname{vwuh}(z)=0$, for all $u, v, w \in U$.

Subtracting (2.23) from (2.24), we have

$[u h(z), w] v[u h(z), w]=0$, for all $u, v, w \in U$

By Corollary 2.2 and using $R$ is 2-torsion free, we obtain

$[u h(z), w]=0$, for all $u, w \in U$.

That is,

$[u, w] h(z)+u[h(z), w]=0$, for all $u, w \in U$.

Replacing $u$ by $2 v u$ in (2.25) and since $R$ is 2torsion free, we get

$v[u, w] h(z)+[v, w] u h(z)+v u[h(z), w]=0$.

Using equation (2.25), we obtain

$[v, w] u h(z)=0$, for all $u, w, v \in U$.

That is, $[U, U] U h(Z \cap U)=(0)$. This completes the proof.

Corollary 2.14. Let $R$ be a 2-torsion free semiprime ring, $U$ a noncentral square-closed Lie ideal of $\mathrm{R}$ and $F, G$ generalized derivations associated to the maps $d, h$ of $R$ respectively. If $F([u, v])= \pm[G(u), G(v)]$, for all $u, v \in U$, then $[U, U] U h(Z \cap U)=(0)$.

Corollary 2.15. Let $R$ be a 2 -torsion free prime ring, $U$ a square-closed Lie ideal of $\mathrm{R}$ and $F, G$ generalized derivations associated to the derivations $d, h$ of $R$ respectively. If $F([u, v])=$ $\pm[G(u), G(v)]$, for all $u, v \in U$, then $h(Z \cap U)=$ (0).

Proof. Using the same arguments in the proof of Theorem 2.13, we get

$[u h(z), w] U[u h(z), w]=0$, for all $u, w \in U$.
By Lemma 2.4, we have $[u h(z), w]=0$, for all $u, w \in U$. Since $h$ is derivation, we get $h(z) \in Z$. We conclude that $[u, w] h(z)=0$, for all $u, w \in U$. Again, using Lemma 2.4, we have $h(Z \cap U)=$ (0).

Corollary 2.16. Let $R$ be a 2-torsion free semiprime ring, $U$ a square-closed Lie ideal of $\mathrm{R}$ and $G$ a multiplicative generalized derivation associated with map $h$ of $R$. If $G$ is SCP on $U$, then $[U, U] U h(Z \cap U)=(0)$.

Proof. Taking $F=I$, where $I$ is an identity mapping, we have $[U, U] U h(Z \cap U)=(0)$. This completes proof.

Theorem 2.17: Let $R$ be a 2-torsion free semiprime ring, $U$ a square-closed Lie ideal of $R$ and $F, G$ multiplicative generalized derivations associated to the maps $d, h$ of $R$ respectively. If $F(u \circ v)= \pm(G(u) \circ G(v))$, for all $u, v \in U$, then $[U, U] U h(Z \cap U)=(0)$.

Proof. By hypothesis, we have

$F(u \circ v)= \pm(G(u) \circ G(v))$, for all $u, v \in U$.

Replacing $v$ by $2 v z, z \in Z \cap U$ in the hypothesis and using this, we have

$0=F(u \circ v z) \pm(G(u) \circ G(v z))$,

$=F((u \circ v) z) \pm(G(u) \circ(G(v) z+v h(z)))$

$=F(u \circ v) z+(u \circ v) d(z) \pm(G(u) \circ G(v)) z \pm$ $(G(u) \circ v) h(z) \mp v[G(u), h(z)]$

$=(u \circ v) d(z) \pm(G(u) \circ v) h(z) \mp v[G(u), h(z)]$

That is

$(u \circ v) d(z) \pm(G(u) \circ v) h(z) \mp v[G(u), h(z)]=$ 0 .

Replacing $v$ by $2 w v, w \in U$ in the last equation, we obtain

$w(u \circ v) d(z)+[u, w] v d(z) \pm w(G(u) \circ$ $v) h(z) \mp[G(u), w] v h(z) \mp w v[G(u), h(z)]=0$.

Using equation (2.26), we get 
$[u, w] v d(z) \mp[G(u), w] v h(z)=0 \quad$ for all $u, v, w \in U$.

This equation is same as (2.21) in the proof of Theorem 2.13. Hence, using the same arguments in there, we get the required results.

Corollary 2.18. Let $R$ be a 2-torsion free semiprime ring, $U$ a noncentral square-closed Lie ideal of $\mathrm{R}$ and $F, G$ generalized derivations associated to the maps $d, h$ of $R$ respectively. If $F(u \circ v)= \pm(G(u) \circ G(v))$, for all $u, v \in U$, then $[U, U] U h(Z \cap U)=(0)$.

Corollary 2.19. Let $R$ be a 2 -torsion free prime ring, $U$ a square-closed Lie ideal of $\mathrm{R}$ and $F, G$ generalized derivations associated to the derivations $d, h$ of $R$ respectively. If $F(u \circ v)=$ $\pm(G(u) \circ G(v))$, for all $u, v \in U$, then $h(Z \cap U)=$ (0).

Acknowledgments: This work is supported by the Scientific Research Project Fund of Cumhuriyet University under the project number F-515.

\section{REFERENCES}

[1]. Ali, A., Yasen, M., and Anwar, M., Strong commutativity preserving mappings on semiprime rings, Bull. Korean Math. Soc., 43(4), 711-713, 2006.

[2]. Ashraf, M., Rehman, N. On derivations and commutativity in prime rings, East-West $\mathrm{J}$. Math. 3(1), 87-91, 2001.

[3]. Ashraf, M., Asma, A. and Shakir, A. Some commutativiy theorems for rings with generalized derivations, Southeast Asain Bull. of Math. 31, 2007, 415-421.

[4]. Awtar, R., Lie structure in prime rings with derivations, Publ. Math. Debrecen , 31, 209215, 1984.

[5]. Bell, H. E., Daif, M. N., On commutativity and strong commutativity preserving maps, Canad. Math. Bull., 37(4), 443-447, 1994.

[6]. Bergen, J., Herstein, I. N. and Kerr, W., Lie ideals and derivation of prime rings, J. of Algebra, 71, 259-267, 1981.
[7]. Bresar, M., On the distance of the compositions of two derivations to the generalized derivations, Glasgow Math. J., 33 (1), 89-93, 1991.

[8]. Daif, M. N., When is a multiplicative derivation additive, Int. J. Math. Math. Sci., 14(3), 615-618, 1991.

[9]. Daif, M. N., Tamman El-Sayiad, M. S., Multiplicative generalized derivation which are additive, East-West J. Math., 9(1), 31-37, 1997.

[10]. Dhara, B., Ali, S., On multiplicative (generalized) derivation in prime and semiprime rings, Aequat. Math., 86, 65-79, 2013.

[11]. Gölbaşı, Ö., Multiplicative generalized derivations on ideals in semiprime rings, Math. Slovaca, 66(6), 2016.

[12]. Goldman, H., Semrl, P., Multiplicative derivations on $C(X)$, Monatsh Math., 121(3), 189-197, 1969.

[13]. Hongan, M., Rehman, N. and Al-Omary, R. M., Lie ideals and Jordan triple derivations in rings, Rend. Semin. Mat. Univ. Padova, 125, 147-156, 2011.

[14]. Martindale III, W. S., When are multiplicative maps additive, Proc. Amer. Math. Soc., 21, 695-698, 1969.

[15]. Posner, E. C., Derivations in prime rings, Proc Amer.Math.Soc., 8, 1093-1100, 1957.

[16]. Rehman, N., Hongan, M., Generalized Jordan derivations on Lie ideals associate with Hochschild 2 -cocycles of rings, Rend. Circ. Mat. Palermo, 60(3), 437-444, 2011

[17]. Tiwari, S. K., Sharma, R. K. and Dhara, B., Multiplicative (generalized)-derivation in semiprime rings, Beitr. Algebra Geom., 1$15,2015$. 